\title{
Active-site structure, binding and redox activity of the heme- thiolate enzyme CYP2D6 immobilized on coated Ag electrodes: a surface-enhanced resonance Raman scattering study
}

\author{
Alois Bonifacio - Diego Millo $\cdot$ Peter H. J. Keizers • \\ Roald Boegschoten · Jan N. M. Commandeur • \\ Nico P. E. Vermeulen · Cees Gooijer · Gert van der Zwan
}

Received: 2 July 2007 / Accepted: 8 September 2007/Published online: 26 September 2007

(c) SBIC 2007

\begin{abstract}
Surface-enhance resonance Raman scattering spectra of the heme-thiolate enzyme cytochrome P450 2D6 (CYP2D6) adsorbed on Ag electrodes coated with 11-mercaptoundecanoic acid (MUA) were obtained in various experimental conditions. An analysis of these spectra, and a comparison between them and the RR spectra of CYP2D6 in solution, indicated that the enzyme's active site retained its nature of six-coordinated low-spin heme upon immobilization. Moreover, the spectral changes detected in the presence of dextromethorphan (a CYP2D6 substrate) and imidazole (an exogenous heme axial ligand) indicated that the immobilized enzyme also preserved its ability to reversibly bind a substrate and form a heme-imidazole complex. The
\end{abstract}

Electronic supplementary material The online version of this article (doi:10.1007/s00775-007-0303-1) contains supplementary material, which is available to authorized users.

A. Bonifacio · D. Millo · C. Gooijer · G. van der Zwan ( $\square)$

Analytical Chemistry and Applied Spectroscopy,

Vrije Universiteit Amsterdam,

De Boelelaan 1083a,

1081 HV Amsterdam,

The Netherlands

e-mail: zwan@few.vu.nl

P. H. J. Keizers - J. N. M. Commandeur - N. P. E. Vermeulen

Molecular Toxicology,

Vrije Universiteit Amsterdam,

De Boelelaan 1083a,

1081 HV Amsterdam,

The Netherlands

R. Boegschoten

Mechanical Workshop,

Vrije Universiteit Amsterdam,

De Boelelaan 1083a,

1081 HV Amsterdam,

The Netherlands reversibility of these processes could be easily verified by flowing alternately solutions of the various compounds and the buffer through a home-built spectroelectrochemical flow cell which contained a sample of immobilized protein, without the need to disassemble the cell between consecutive spectral data acquisitions. Despite immobilized CYP2D6 being effectively reduced by a sodium dithionite solution, electrochemical reduction via the Ag electrode was not able to completely reduce the enzyme, and led to its extensive inactivation. This behavior indicated that although the enzyme's ability to exchange electrons is not altered by immobilization per se, MUA-coated electrodes are not suited to perform direct electrochemistry of CYP2D6.

Keywords Surface-enhanced resonance Raman scattering - Cytochrome P450 2D6 .

Enzyme immobilization $\cdot \mathrm{Ag}$ electrodes

$\begin{array}{ll}\text { Abbreviations } \\ \text { 5cHS } & \text { Five-coordinated high spin } \\ \text { 6cLS } & \text { Six-coordinated low spin } \\ \text { CYP } & \text { Cytochrome P450 } \\ \text { DX } & \text { Dextromethorphan } \\ \text { MUA } & \text { 11-Mercaptoundecanoic acid } \\ \text { MV } & \text { Methyl viologen } \\ \text { NTA } & \text { Nitrilotriacetic acid } \\ \text { RR } & \text { Resonance Raman } \\ \text { SAM } & \text { Self-assembled monolayer } \\ \text { SERRS } & \text { Surface-enhanced resonance Raman scattering } \\ & \\ \text { Introduction } \\ \text { Heme proteins are a large class of proteins having a } \\ \text { substituted iron porphyrin as a cofactor, with functions }\end{array}$


ranging from electron carriers to oxygen transport to catalysis. Heme enzymes such as cytochromes P450 (CYPs) and other oxygenases, being able to bind several substrates and to use electrons from their heme iron to drive a catalytic reaction, seem to be promising for nanobiotechnological applications in biosensing and biocatalysis [1,2].

The immobilization of enzymes on solid supports or electrodes is a commonly used approach for constructing biosensors or biocatalytic devices [3, 4]. In principle, electrodes can be used as a source of electrons to drive a catalytic reaction, or as elements of biosensors which translate a molecular event such as substrate binding into a measurable electric signal.

The question arises whether immobilization preserves the protein function or rather induces losses of enzymatic activity, and what structural changes cause the dysfunction [1]. Therefore, techniques capable of probing the activity and the structure of enzymes immobilized on surfaces are valuable.

Surface-enhanced resonance Raman scattering (SERRS) has proven to be a sensitive and selective spectroscopic technique to study heme proteins immobilized on metal surfaces, and it has been used to characterize the active site of proteins such as cytochromes $c$, cytochrome $c$ oxidase and CYPs [5]. This technique is based on the inelastic scattering of laser radiation whose wavelength is in resonance with an electronic transition of the molecule studied [6-8], leading to a signal enhancement denoted as the resonance Raman (RR) effect. In the case of proteins having a heme as a chromophore, the porphyrin vibrational modes are intensified over the other protein vibrations if the laser wavelength falls within an associated electronic absorption band. The heme moiety can thus be selectively investigated without a dominating interference due to the protein matrix. Additional sensitivity is gained through surface enhancement (or surface-enhanced Raman scattering effect) if the protein is close to a metal surface with particular characteristics. Depending on the molecule studied and on the experimental conditions applied, the synergy between the two signal-enhancing effects (i.e., SERRS) sometimes reaches single-molecule sensitivity [9-12], therefore allowing the study of enzymes immobilized on surfaces, typically consisting of very low amounts of sample (i.e., from a few layers to submonolayers of protein).

To fulfill the requirements to achieve both resonance enhancement and surface enhancement, silver is the surfaceenhancing metal of choice [7]. Recently, heme proteins such as cytochromes $c$ [5, 13-19] and CYP101 [20] have been investigated using SERRS on roughened $\mathrm{Ag}$ electrodes coated with self-assembled monolayers (SAMs) of several different alkanethiols and their derivatives. SAMs of functionalized alkanethiols are among the most versatile coatings for both electrostatic and covalent immobilization of pro- teins to metal surfaces, having the additional advantage of preventing possible negative consequences of the direct contact between the protein and the metal [5, 21]. Besides technological applications, SERRS on SAM-coated electrodes can become an important tool in fundamental studies about protein-membrane and protein-protein interactions. In fact, because of their structure and characteristics, SAMs can be thought of as a rough model for biological membranes $[5,13]$, or can be used to mimic the binding domain of a partner protein, since surface properties such as polarity and charge can be selected by choosing the appropriate alkanethiol derivative [13, 22]. Moreover, electrochemically roughened Ag electrodes coated with SAMs were recently shown to be excellent for studying immobilized proteins with voltammetric techniques, so both SERRS and voltammetry can be performed on the same sample [19].

In the work reported here, we exploited SERRS to study the behavior of the human enzyme CYP2D6 electrostatically immobilized on a $\mathrm{Ag}$ electrode coated with a SAM of 11-mercaptoundecanoic acid (MUA). CYPs are hemethiolate monooxygenases capable of oxidizing and reducing a broad range of endogenous and exogenous substrates and can be found in all kinds of tissues in virtually all organisms [23]. CYP2D6 is a peripheral membrane-bound protein and is one of the most important CYPs in humans, being involved in the metabolism of about $30 \%$ of the currently marketed drugs, including $\beta$-blockers, neuroleptics, antidepressants and antiarythmics [24, 25]. Its characteristics make this enzyme not only attractive for technological applications as a biocatalyst or biosensor, but also from a toxicological and clinical point of view. In fact, a better understanding of its mechanisms of catalysis could help in developing more efficient drugs [26, 27].

Using a home-built SERRS spectroelectrochemical flow cell [28], we investigated the CYP2D6 active-site structure under diverse experimental conditions in order to check the functionality of the immobilized enzyme, its redox activity and its ability to bind reversibly a substrate or an exogenous heme ligand such as imidazole. Besides confirming the retention of the enzyme's function upon immobilization, SERRS spectra yield information about structural changes in the active site associated with substrate binding and electron transfer, the initial two steps of a CYP catalytic cycle.

\section{Materials and methods}

\section{Chemicals}

The pSP19T7LT plasmid containing bicistronically human CYP2D6 with a C-terminal His $_{6}$-tag and human NADPHCYP reductase, was kindly provided by Magnus IngelmanSundberg. Dextromethorphan (DX), imidazole, MUA and 
sodium dithionite were purchased from Sigma-Aldrich (St Louis, USA). Emulgen 911 was obtained from KAO Chemicals (Tokyo, Japan). Nickel nitrilotriacetic acid (NTA) agarose was from Sigma. Phosphate buffer solutions $\left(\mathrm{pH} 7.4,40 \mathrm{mM}\right.$ ) were prepared from $\mathrm{K}_{2} \mathrm{HPO}_{4}$ and $\mathrm{KH}_{2} \mathrm{PO}_{4}$ (J.T. Baker, Deventer, The Netherlands). All other chemicals were of analytical grade and were obtained from standard suppliers.

\section{Expression and purification of CYP2D6}

The pSP19T7LT plasmids containing wild-type CYP2D6 were transformed in Escherichia coli, strain JM109. Expression, membrane isolation and purification were carried out as previously described [29]. In short, membranes were resuspended in $0.5 \%$ of the original culture volume of potassium phosphate-glycerol buffer $(50 \mathrm{mM}$ potassium phosphate buffer, $\mathrm{pH} 7.4,10 \%$ glycerol) and CYP contents were determined by $\mathrm{CO}$ difference spectra [30]. The enzyme was solubilized by stirring in potassium phosphate-glycerol supplemented with 0.5\% Emulgen 911 for $2 \mathrm{~h}$ at $4{ }^{\circ} \mathrm{C}$. Insoluble parts were removed by centrifugation $\left(60 \mathrm{~min}, 120,000 \mathrm{~g}\right.$ at $\left.4{ }^{\circ} \mathrm{C}\right)$. The supernatant was mixed, while gently rocking it, with nickel nitrilotriacetic acid agarose for $60 \mathrm{~min}$ at $4{ }^{\circ} \mathrm{C}$. The nickel nitrilotriacetic acid agarose was then applied to a polypropylene tube with a porous disk (Pierce, Perbio Science, Etten-Leur, The Netherlands), washed with potassium phosphate-glycerol buffer containing $2 \mathrm{mM}$ histidine. Subsequently, bound CYP2D6 was eluted with $0.2 \mathrm{M}$ histidine. After overnight dialysis in potassium phosphate-glycerol buffer the sample was concentrated on a Vivaspin 20 filtration tube $(10,000$ MWCO PES, Sartorius, Nieuwegein, The Netherlands) to a final concentration of $60 \mu \mathrm{M}$ CYP2D6.

\section{Spectroelectrochemical flow cell}

SERRS measurements were performed using a modified version of a linearly moving low-volume spectroelectrochemical cell previously described [28]. The cell was originally designed to acquire potential-dependent SERRS spectra from proteins adsorbed on roughened $\mathrm{Ag}$ electrodes using a Raman microscope in a backscattering configuration. The modified version of this cell used in the present work has the possibility to flow a solution through the cell via two small holes (0.75-mm diameter each; Fig. 1). By using a valve, a liquid-chromatography pump (Gilson, Middleton, USA) and flexible PEEK tubing (VICI International, Schenkon, Switzerland), the thin layer $(0.3 \mathrm{~mm})$ of solution in contact with the $\mathrm{Ag}$ electrode surface can be changed by flowing different solutions though the spectroelectrochemical cell, without the need to disassemble the cell itself (Fig. 1). Moreover, by degassing the solutions with argon and by using oxygen-proof PEEK tubing, the presence of oxygen, which can interfere with electrochemical and spectroscopic measurements, can be minimized in the cell. It should be noted that small internal diameter of the liquid-chromatography tubing $(0.75 \mathrm{~mm})$ and the low internal volume of the cell (approximately $50 \mu \mathrm{L}$ ) allows the use of relatively small amounts of solutions.

\section{Raman spectroscopy instrumentation}

Spectroscopic measurements were conducted by placing the spectroelectrochemical cell under a home-built Raman microscope in a backscattering configuration: a Zeiss microscope (D-7082 with a $\times 40$ objective, numerical aperture 0.60 , working distance $2 \mathrm{~mm}$ ) was coupled to an Andor Shamrock SR-303i-A single monochromator (Andor Technologies DV-420OE, Belfast, UK) with a mounted $2,400 \mathrm{~g} \mathrm{~mm}^{-1}$ holographic grating and an Andor Newton DU970N CCD camera. The $413.1 \mathrm{~nm}$ line of a continuouswave $\mathrm{Kr}$ ion laser (Innova 300C, Coherent, Santa Clara, CA, USA) was used for excitation. The Rayleigh scattered light was removed using third millennium edge long pass filter. Laser powers of $5 \mathrm{~mW}$ at the sample and an accumulation time of $180 \mathrm{~s}$ were used throughout the experiments. To avoid sample photodegradation, the cell was put in a moving holder which ensured a constant linear movement of the metal electrode surface under the microscope objective, while keeping the focus approximately constant [28]. The monochromator slit was set to $120 \mu \mathrm{m}$, yielding a resolution of approximately $4 \mathrm{~cm}^{-1}$, with an increment of approximately $0.8 \mathrm{~cm}^{-1}$ per data point.

The sloping background of the spectra was subtracted using a baseline fitted to the experimental data with the Andor CCD camera software, while the fitting of the CYP2D6 spectrum at $-1.0 \mathrm{~V}$ with Lorentzian functions was performed with PeakFit ${ }^{\circledR}$ version 4.12 (SeaSolve Software, Richmond, CA, USA).

\section{Electrochemical instrumentation}

Polycrystalline silver disk electrodes (IJ Cambria Scientific, Burry Port, UK) of 2-mm diameter (geometric area $0.0314 \mathrm{~cm}^{2}$ ) were used as working electrodes; a platinum ring and a saturated calomel electrode (AMEL Instruments, Milan, Italy), were used as the counter electrode and the reference electrode, respectively. The reference electrode was kept in a glass tube filled with a $40 \mathrm{mM}$ potassium phosphate buffer solution and separated from the working solution by a PEEK porous frit. It was 


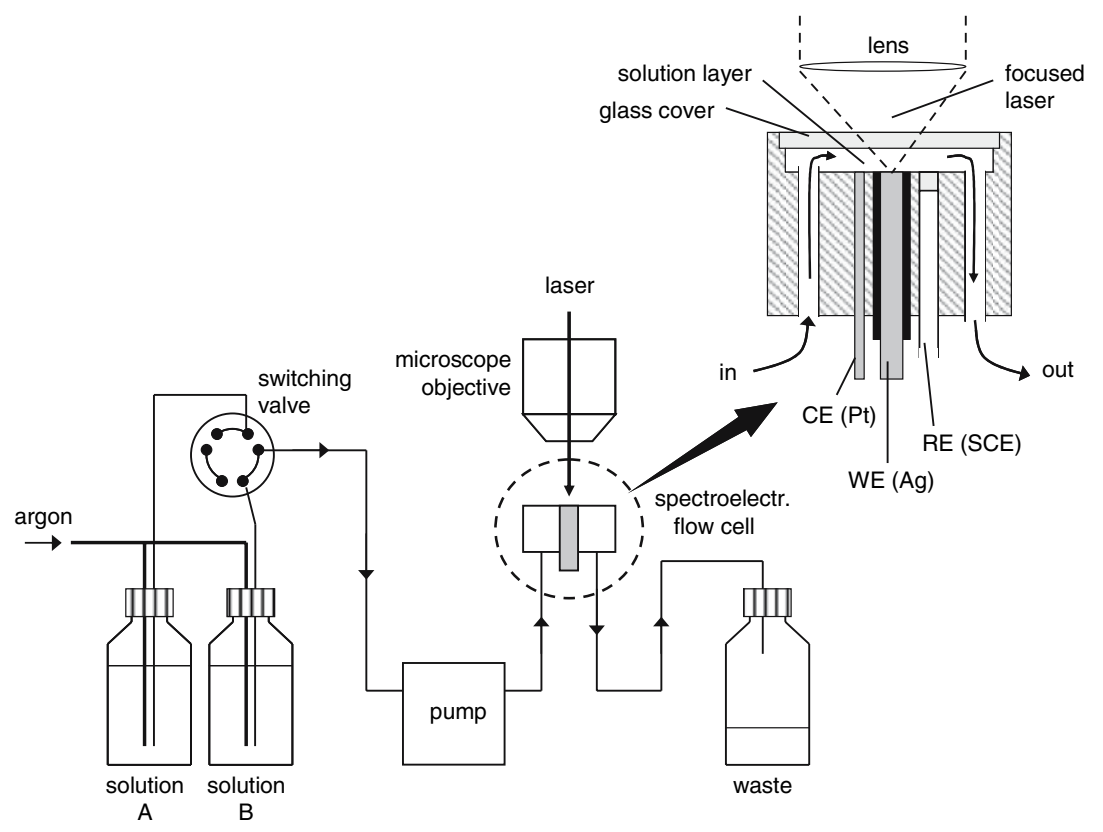

Fig. 1 The surface-enhanced resonance Raman scattering (SERRS) spectroelectrochemical flow system. The degassed solutions are pumped though PEEK liquid-chromatography tubing into the cell and then to the waste. A switching valve is used to select which solution has to be pumped. A scheme of the cell is shown in detail, with the inlet and outlet for the flowing solution and the three

kept at constant room temperature $\left(20 \pm 0.1{ }^{\circ} \mathrm{C}\right)$ during all the experiments. The three-electrode system was controlled with a $\mu$ Autolab potentiostat (Eco Chemie, Utrecht, The Netherlands). All potentials are referred to a saturated calomel electrode, previously calibrated against the methyl viologen (MV) $\mathrm{MV}^{2+} / \mathrm{MV}^{+}$couple.

\section{Sample preparation}

SERRS measurements were performed on electrochemically roughened Ag electrodes coated with MUA, prepared as follows. Ag electrodes were treated as previously described [31] with water on aluminum oxide lapping film sheets $\left(261 \times\right.$ and $\left.262 \times, 3 \mathrm{M}^{\mathrm{TM}}\right)$ from 5 - to $1-\mu \mathrm{m}$ grain size until a mirrorlike appearance of the surfaces was obtained. After they had been polished, the electrodes were roughened ex situ with an oxidation-reduction cycle procedure similar to that described by Roth et al. [32] and then kept overnight in $2 \mathrm{mM}$ ethanol solution of MUA. The oxidation-reduction cycles (approximately $0.67 \mathrm{C} \mathrm{cm}^{-2}$ of anodic charge passed per step) were performed in an ordinary glass electrochemical cell $(5 \mathrm{~mL})$ filled with a $0.1 \mathrm{M} \mathrm{KCl}$ solution using the three-electrode system described already.

The MUA-coated Ag electrodes were rinsed with ethanol to eliminate the excess of MUA, dried by gently electrodes: the $\mathrm{Ag}$ working electrode $(W E)$, the Pt counter electrode $(C E)$ and the connection though a porous frit to a saturated calomel electrode $(S C E)$ reference electrode $(R E)$. The laser for Raman excitation is focused by the microscope objective onto the $\mathrm{Ag}$ electrode through a glass cover and a layer of solution

blowing nitrogen on them and then immersed into $50 \mu \mathrm{L}$ of a $3 \mu \mathrm{M}$ solution of protein in potassium phosphate buffer (40 mM, pH 7.4, $10 \%$ glycerol) for at least $3 \mathrm{~h}$ at $4{ }^{\circ} \mathrm{C}$, to induce adsorption of the protein on the electrode. Then, the coated electrodes with the adsorbed protein were rinsed with buffer to eliminate possible excess of loosely bound protein, and were put into the spectroelectrochemical cell described already.

Depending on the required experimental conditions, before every SERRS measurement the appropriate solution was flowed for 1-2 min into the cell: for measurements in the absence of DX or imidazole, a potassium phosphate buffer (40 mM, pH 7.4) was used, while for measurements in the presence of DX or imidazole, solutions of DX $(5 \mathrm{mM}, 0.02 \%$ of methanol) or imidazole $(10 \mathrm{mM})$ in buffer were used. The solutions of DX were prepared by diluting with buffer a $2 \mathrm{M}$ stock solution of DX in methanol. To chemically reduce the protein in the absence or presence of substrate, sodium dithionite solutions $(5 \mathrm{mg}$ $\mathrm{mL}^{-1}$ ) were prepared using buffer solutions or DX solutions $(5 \mathrm{mM})$ in buffer, and were flowed for 1-2 min into the cell before every SERRS measurement. Prior to use, all the solutions flowed into the cell were deoxygenated for at least $3 \mathrm{~h}$ with argon.

RR measurements were performed using buffered CYP2D6 solutions in spinning glass capillaries as previously described [33]. To chemically reduce the enzyme in 
solution prior to RR measurements, solid sodium dithionite (to a final concentration of $5 \mathrm{mg} \mathrm{mL}^{-1}$ ) was added to a buffered 3-5 $\mu \mathrm{M}$ protein solution in presence of $5 \mathrm{mM}$ DX, previously degassed with argon.

\section{Results and discussion}

The resting state

Intense SERRS spectra (Fig. 2, spectra a) were observed from CYP2D6 immobilized via electrostatic interaction [34] on MUA-coated Ag electrodes at open circuit (i.e.. no external potential is applied), in the presence of phosphate buffer. These experimental conditions should reproduce the "resting state" of the enzyme (i.e.. the starting point of its catalytic cycle) when no substrate is present in the active site and no reaction is taking place $[35,36]$. A MUA-SAM was chosen as the coating because of its structural stability, its compatibility with CYP2D6 [34] and its use in recent SERRS studies on bacterial CYP101 [20] and on cytochrome $c[5,13]$.

The most intense band of the SERRS spectra is observed at $1,371 \mathrm{~cm}^{-1}$, and a group of bands are detected between 1,450 and $1,650 \mathrm{~cm}^{-1}$. The frequencies of the vibrational modes above $1,300 \mathrm{~cm}^{-1}$ are correlated with the oxidation, the spin and the coordination state of the iron atom and therefore they are referred to as "marker bands" for such properties [37]. The most relevant marker bands in CYP2D6 SERRS spectra are assigned to the corresponding vibrational modes on the basis of former RR studies of CYP2D6 [33] and other CYPs [37] in buffered solution (Table 1). According to the frequencies observed, the heme iron in the enzyme active site is oxidized $\left(\mathrm{Fe}^{3+}\right)$, mainly six-coordinated low-spin (6cLS) state, as is usually expected from a CYP in its resting state [36, 37]. An extensive amount of experimental data from other CYPs as well as theoretical studies showed that in the $6 \mathrm{cLS}$ state the $\mathrm{Fe}^{3+}$ atom has the four pyrrolic nitrogens of the porphyrin macrocycle as equatorial ligands, and a cysteine and a water molecule (or hydroxyl ion) as axial ligands [35, 36].

The observation of an oxidized 6cLS state is in agreement with spectroscopic data on CYP2D6 previously reported. In particular, the SERRS spectrum of CYP2D6 under these conditions is identical to the RR spectrum of the same enzyme in buffer [33, 38] (Fig. 3 spectra b and $\left.b^{\prime}\right)$. An analogous correspondence with RR data was recently reported for SERRS spectra of CYP2D6 adsorbed on Ag nanoparticles coated with MUA [34]. This striking

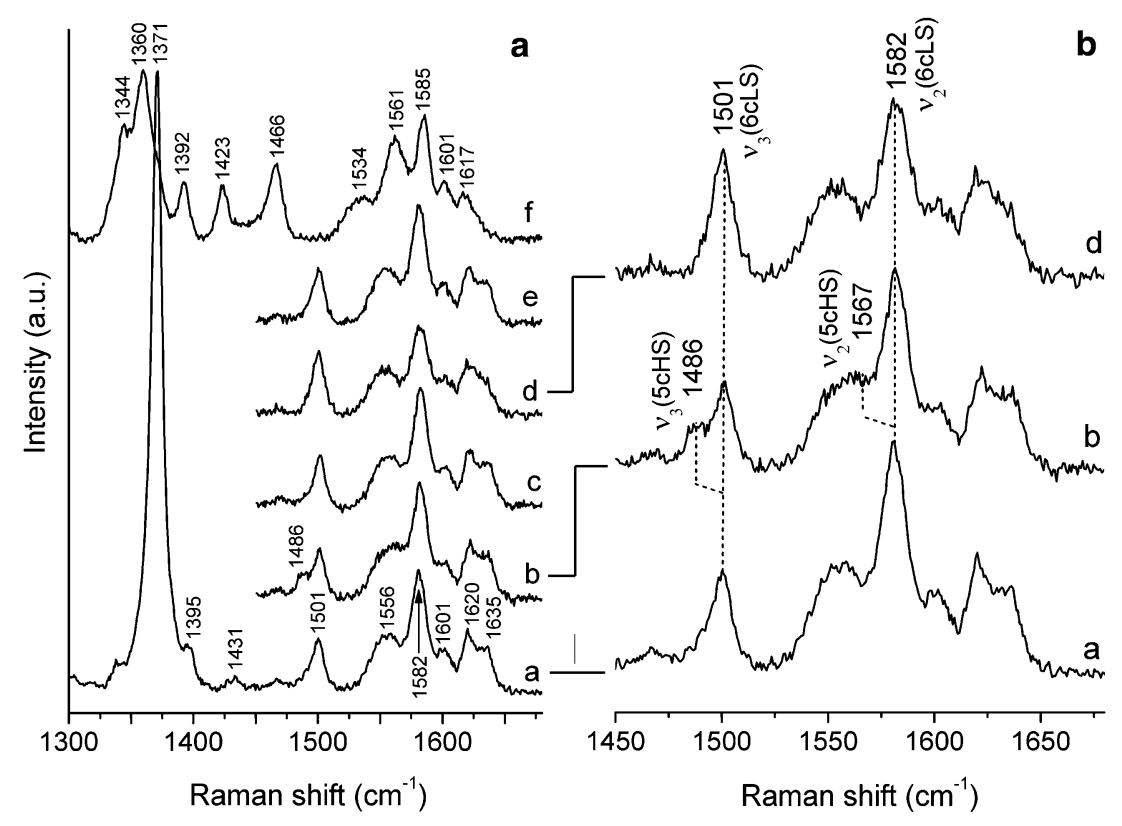

Fig. 2 A SERRS spectra of cytochrome P450 (CYP) 2D6 on an 11mercaptoundecanoic acid (MUA)-coated Ag electrode obtained by consecutively flowing different solutions in the spectroelectrochemical cell (at open circuit), in the following order: $a$ buffer, $b$ dextromethorphan (DX) buffered solution, $c$ buffer (rinse), $d$ imidazole-buffered solution, $e$ buffer (second rinse), $f$ dithionite and DX solution in phosphate buffer. The $1,300-1,450-\mathrm{cm}^{-1}$ regions of spectra $b-e$ are identical to that of spectrum $a$ and are omitted for visual clarity. $B$ Detailed view of the $1,450-1,660-\mathrm{cm}^{-1}$ region of the CYP2D6 SERRS spectra in presence of $a$ buffer, $b$ DX and $d$ imidazole. Bands corresponding to the $v_{2}$ and $v_{3}$ vibrational modes for the six-coordinated low-spin and five-coordinated high-spin heme species are indicated. Experimental details are given in "Materials and methods" 
Table 1 Vibrational frequencies $\left(\mathrm{cm}^{-1}\right)$ and relative intensities of most relevant oxidation- $\left(v_{4}\right)$ and spin/coordination- $\left(v_{3}, v_{2}\right)$ marker bands in SERR spectra of CYP2D6 adsorbed on MUA-coated electrodes, under different experimental conditions

\begin{tabular}{|c|c|c|c|c|}
\hline \multirow[t]{3}{*}{ Mode } & \multicolumn{3}{|l|}{$\mathrm{Fe}^{3+}$} & \multirow{3}{*}{$\begin{array}{l}\mathrm{Fe}^{2+} \\
5 \mathrm{cHS}^{\mathrm{a}}\end{array}$} \\
\hline & \multicolumn{2}{|l|}{$6 \mathrm{cLS}$} & \multirow{2}{*}{$\begin{array}{l}6 \mathrm{cLS} / 5 \mathrm{cHS} \\
\text { With substrate (dextromethorphan) }\end{array}$} & \\
\hline & Without substrate/inhibitor & With inhibitor (imidazole) & & \\
\hline$v_{2}$ & $1,582(0.19)$ & $1,582(0.14)$ & $1,582(0.20) / 1,567(\mathrm{NA})^{\mathrm{b}}$ & $1,561(0.91)$ \\
\hline$v_{3}$ & $1,501(0.08)$ & $1,501(0.10)$ & $1,501(0.09) / 1,486(0.05)$ & $1,466(0.65)$ \\
\hline$v_{4}$ & $1,371(1.00)$ & $1,371(1.00)$ & $1,371(1.00)$ & $1,344^{\mathrm{c}}(1.00)$ \\
\hline
\end{tabular}

Relative intensities are reported after the corresponding frequencies between parentheses; values are relative to the band with an intensity of 1.00 $6 c L S$ six-coordinated low spin, $5 c H S$ five-coordinated high spin, $N A$ not available

${ }^{\text {a }}$ Reduced state is achieved by adding dithionite in presence of substrate

b Band not sufficiently resolved, frequency estimated by spectral difference [33]

c Together with this band, in spectra of CYP2D6 another one is observed at $1,360 \mathrm{~cm}^{-1}$, characteristic of reduced inactive cytochrome P420 species

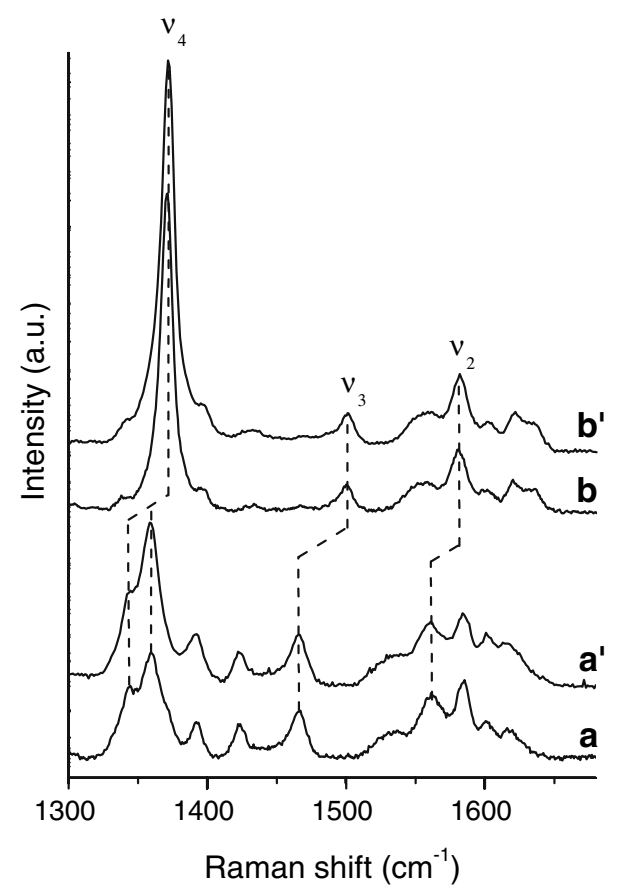

Fig. 3 Comparison between SERRS $(a, b)$ and resonance Raman $\left(a^{\prime}\right.$, $\left.b^{\prime}\right)$ spectra of reduced $\left(a, a^{\prime}\right)$ and oxidized $\left(b, b^{\prime}\right)$ CYP2D6. SERRS spectra were obtained from MUA-coated $\mathrm{Ag}$ electrodes in the presence of buffer alone $(b)$ and a dithionite and DX solution in phosphate buffer $(a)$, while resonance Raman spectra were obtained from CYP2D6 in solution under the same conditions. Marker bands for oxidation $\left(v_{4}\right)$ and spin $\left(v_{3}, v_{2}\right)$ states are indicated. Experimental details are given in "Materials and methods"

similarity in the position and relative intensity of SERRS and RR spectra suggests that, differently from what was recently reported for CYP101 [20], CYP2D6 retains its active-site structure upon adsorption on the SAM-coated electrode, or at least that there are no structural changes inducing significant alterations in heme properties.
Reversible binding of a substrate

Although the active-site structure is likely to be unaffected by the protein-surface interaction, the functioning of the adsorbed enzyme still has to be verified. According to the accepted scheme of the catalytic cycle common to most CYPs, the heme iron may experience changes in its coordination sphere and its spin as a consequence of the presence of substrates in the active site [36]. RR spectra of CYP2D6 in the presence of DX, a substrate of this enzyme, showed the appearance of marker bands for a five-coordinated high-spin (5cHS) heme besides the 6cLS heme already present in the resting state [33]. In agreement with those RR data, SERRS spectra of CYP2D6 adsorbed on coated electrodes, upon flowing a DX solution into the cell (Fig. 2, spectra b), exhibit distinct differences from the spectra of the enzyme in the resting state, showing additional bands characteristic of a $5 \mathrm{cHS}$ heme $\left(v_{3}\right.$ at $1,486 \mathrm{~cm}^{-1}$ and $v_{2}$ at $1,567 \mathrm{~cm}^{-1}$; Fig. 2, spectra $b$, Table 1).

This partial transition from 6cLS to 5cHS is explained by the displacement of a water molecule as the heme's distal axial ligand by DX, which is accommodated in the active site on the distal side above the porphyrin macrocycle [33]. Water molecules, which may still be present in the active site, can then rebind to the heme iron, yielding an equilibrium between the 5cHS and 6cLS states [33, 37, 39].

The spectral changes taking place owing to the presence of DX therefore clearly indicate that the adsorbed enzyme retains its ability to accommodate a substrate into its active site, confirming similar results reported for CYP2D6 adsorbed on coated $\mathrm{Ag}$ nanoparticles [34]. Unfortunately, neither RR nor nanoparticle-based SERRS allowed the investigation of the reversibility of the substrate-binding process, since it is extremely difficult to rapidly eliminate the substrate from the enzyme-containing sample. Such problems are not encountered in the 
present spectroelectrochemical cell setup. The substrate is easily removed from the enzyme-coated electrode surface by flowing buffer through the cell, reestablishing the resting state conditions. Once the buffer had completely replaced the DX solution in the cell, 5cHS marker bands disappeared from SERRS spectra of CYP2D6, which recover the shape characteristic of the $6 \mathrm{cLS}$ heme in the resting state (Fig. 2, spectrum c). The exchange between the two solutions can be operated many times, showing every time the concomitant spectral changes, and indicating an intrinsic stability of the CYP2D6-SAMelectrode system. The similarity between the SERRS spectra of the same enzyme sample before the introduction of DX (Fig. 2, spectra a) and after its removal (Fig. 2, spectrum c) clearly demonstrates that the adsorbed CYP2D6 is able to reversibly bind a substrate, implying that the interaction with the coated electrode surface does not obstruct the substrate access routes to the enzyme active site.

Reversible binding of imidazole as an exogenous heme axial ligand

Imidazole and several imidazole derivatives are believed to form a relatively strong bond via their nitrogen with the heme iron of ferric CYPs, replacing the water molecule as a distal axial ligand, and thereby preventing the binding of oxygen to the heme in a subsequent step of the catalytic cycle [37, 40-43]. Since oxygen binding is essential for monooxygenase activity, often these compounds are effective inhibitors of CYPs [44].

The SERRS spectra of CYP2D6 collected upon flowing an imidazole solution over the enzyme-coated electrode are shown in Fig. 2, spectra d, displaying marker bands characteristic of a 6cLS state (Table 1), as expected from a CYP heme having an imidazole as the sixth axial ligand. Although the frequencies of the imidazole complex are identical to those reported for the resting state, the relative intensity ratio between the $v_{3}$ and $v_{2}$ bands is visibly increased with respect to the 6cLS resting state from 0.4 to 0.7 (Fig. 2spectra a and d, Table 1), in agreement with RR data of CYP2D6 in solution (see supplementary material) and with RR spectra previously reported for other CYPimidazole complexes [41, 45-47]. This difference in relative intensities has been observed in all the RR spectra of CYP-imidazole complexes reported so far, and is generally regarded as the characteristic spectral feature which allows one to discriminate between the two 6cLS states having either imidazole or water as the heme axial ligand in CYPs. The explanation given for these characteristic relative intensity changes observed in the presence of imidazole in RR spectra of CYPs invokes the different resonance conditions due to redshift of the Soret absorption induced by imidazole in CYPs [37].

An additional but less pronounced feature observed in the SERRS spectrum of the CYP2D6-imidazole complex is the intensity decrease in the region around $1,630 \mathrm{~cm}^{-1}$, where three overlapping bands are expected: two stretching modes of the heme vinyl substituents around 1,620 and 1,630 $\mathrm{cm}^{-1}$, and the $v_{10}$ porphyrin mode around $1,635 \mathrm{~cm}^{-1}$ [37]. The presence of another vinyl stretching band, otherwise invisible because of its overlap with the more intense neighboring bands, can be substantiated by fitting the data with Lorentzian functions. A qualitative analysis of the fitting supports the hypothesis of a decrease in relative intensity of a vinyl band around $1,630 \mathrm{~cm}^{-1}$ for the imidazole complex (see supplementary material), suggesting a possible perturbation of the vinyls upon imidazole binding [48].

The observed change in relative intensity could be due to a variation in the rotational conformation of the vinyls [48]. Such variation might also cause a shift in the stretching frequencies of the vinyls [49, 50], although in SERRS spectra of CYP2D6 no significant shifts are detected. On the other hand, similar changes in relative intensities in the $1,610-1,640-\mathrm{cm}^{-1}$ spectral region have been previously reported for the imidazole complexes of bacterial and microsomal CYPs [41, 45-47]. The fact that imidazole binding induces similar changes in vinyl rotational conformation in enzymes having rather different active-site structures is somewhat surprising. Since vinyl stretching modes in SERRS spectra are thought to be enhanced via an electronic coupling with the porphyrin [37], an alternative explanation of the changes detected upon imidazole binding could be the difference in resonance conditions due to the shift of the Soret band, similarly to what has been proposed for the $v_{3} / v_{2}$ intensity ratio. However, in absence of further experimental data from other techniques, the SERRS spectra presented cannot reliably give more detailed structural information about conformational changes of vinyls occurring upon imidazole binding.

Similarly to what was observed for DX binding, SERRS spectra obtained by flowing buffer through the cell in place of the imidazole solution (Fig. 2, spectrum e) have the $v_{3} / v_{2}$ relative intensity ratio restored to 0.4 , and are identical to the spectra of the enzyme in the resting state (Fig. 2, spectra a), including the relative intensity pattern around $1,630 \mathrm{~cm}^{-1}$. Therefore, despite the imidazole being expected to form a relatively strong bond with the heme iron, the nonequilibrium situation due to the constant flow of buffer over the CYP-imidazole complex eventually causes the complete dissociation of the imidazole from the enzyme and the concomitant restoration of a water molecule as sixth ligand, demonstrating that the CYP-imidazole complex formation is reversible. 


\section{Redox activity}

The catalytic activity of CYP2D6 relies on its ability to receive electrons from NADPH-dependent CYP reductase to drive the catalysis and to activate molecular oxygen to oxidize the substrate [36]. In particular, the first of the two electrons transferred during a catalytic cycle reduces the iron from $\mathrm{Fe}^{3+}$ to $\mathrm{Fe}^{2+}$, facilitating oxygen binding to the ferrous heme. In their resting state, CYPs usually have a formal reduction potential $\left(E^{\circ \prime}\right)$ lower than that of their electron-donor protein, so heme reduction does not occur [36]. However, the $E^{\circ \prime}$ of some CYPs, including CYP2D6, is shifted to more positive values by the presence of a substrate in the active site, facilitating the reduction of the enzyme heme by the redox partner [1, 36, 51]. Therefore, the electron transfer reaction is believed to be triggered by substrate binding and is usually considered the third step of the catalytic cycle [36].

In absence of reductase, immobilized CYP2D6 might receive electrons from a reducing agent present in solution or directly from the electrode, by applying a proper voltage. The electron transfer process from the alternative electron source to the enzyme can be monitored by examining the relative presence of reduced heme species in SERRS spectra, using solutions previously deoxygenated with argon. This requirement is crucial since dioxygen rapidly binds to the ferrous heme to form a complex, which upon reaction with another electron quickly turns into an instable peroxo-ferric intermediate, continuing the catalytic cycle and hampering the observation of reduced species [36].

Upon flowing over the adsorbed enzyme a deoxygenated and buffered DX solution containing sodium dithionite as a reducing agent, the SERRS spectrum shown in Fig. 2, spectrum $\mathrm{f}$ is observed. It mainly presents bands with frequencies characteristic of a reduced $5 \mathrm{cHS}$ heme (Table 1), which are markedly different from all the spectra of oxidized CYP2D6 obtained so far (Fig. 2, spectra a-e). A noticeable feature is the coexistence of two $v_{4}$ oxidation marker bands at 1,344 and $1,360 \mathrm{~cm}^{-1}$, originating from the downshift and the splitting of the corresponding $v_{4}$ band observed at $1,371 \mathrm{~cm}^{-1}$ in the oxidized enzyme, and indicating the presence of two different heme species. While the $v_{4}$ band at $1,344 \mathrm{~cm}^{-1}$ - consistent with the other bands observed-is typical of a ferrous CYP in its active form, the band at $1,360 \mathrm{~cm}^{-1}$ is characteristic of a ferrous inactive form called P420 [20, 37, 52, 53]. This strongly suggests that upon reduction a significant fraction of the immobilized enzyme undergoes structural changes leading to its inactivation. However, according to the RR spectra of the ferrous active and inactive forms previously reported for other CYPs, the active enzyme still contributes to a significant extent to the observed CYP2D6 SERRS spectrum, whereas no marker bands for the inactive species other than the one at $1,360 \mathrm{~cm}^{-1}$ can be detected. In fact, in all the RR or SERRS spectra of P420 species reported so far for various CYPs, the $v_{4}$ band is the strongest, with relative intensity much higher than for the other bands, whereas in spectra of active enzymes the intensity pattern is different, with many other bands having intensities comparable to that of the $v_{4}$ band [20, 37, 52-55]. In the spectra of reduced CYP2D6, the bands in the 1,400-1,650$\mathrm{cm}^{-1}$ region have relative intensities comparable to that of the $v_{4}$ band of the active enzyme (at $1,344 \mathrm{~cm}^{-1}$ ), whereas the bands of the $\mathrm{P} 420$ species are likely to have relative intensities much smaller than that of the P420 $v_{4}$ band at $1,360 \mathrm{~cm}^{-1}$, and are therefore buried under the more intense bands due to the active enzyme.

Notably, this partial inactivation is not due to the immobilization as such, since every attempt to obtain reduced free CYP2D6 in solution by dithionite addition led to samples containing inactive enzyme besides the active one, yielding RR spectra which closely resemble the SERRS spectrum of the adsorbed enzyme (Fig. 4, spectra a). The inactivation might be due to laser-induced photodegradation, since laser illumination is common to both RR and SERRS. However, the use of laser powers lower than $5 \mathrm{~mW}$ yielded identical spectra (data not shown), and small amounts of inactive enzyme were previously detected in dithionite-reduced CYP2D6 samples using nonlaser techniques as well, such as UV-vis electronic absorption spectroscopy [38].

When reduction of CYP2D6 with dithionite is attempted in the absence of a substrate, the resulting SERRS spectrum presents some differences with respect to that of the substrate-bound enzyme (Fig. 4). In particular, the presence of DX in the dithionite solution increases the amount of reduced active enzyme at the expense of the oxidized and reduced inactive forms, as deduced from the increase in intensity of the band at $1,344 \mathrm{~cm}^{-1}$ and the concomitant decrease of the bands at 1,360 and $1,371 \mathrm{~cm}^{-1}$. Thus, the presence of DX in the heme pocket appears to affect the redox activity of the enzyme, possibly by causing a shift of its $E^{\circ /}$ to more positive values, as previously observed for CYP2D6 on a polyaniline-doped glassy carbon electrode in the presence of fluoxetine [51].

On the other hand, in the electrochemical approach the control of the CYP2D6 oxidation state is attempted by applying a constant potential to the Ag working electrode, during which a SERRS spectrum is recorded to monitor the relative abundance of oxidized and reduced species characteristic for that voltage. Complete oxidation or reduction of the protein should in principle be obtained by applying a potential which is sufficiently more positive or more negative with respect to its $E^{\circ}$, respectively.

Besides the advantage of controlling the enzyme oxidation state and therefore, under adequate conditions, of 
supplying the electrons and driving the catalytic reaction, an additional benefit of this approach is the possibility to study the electron transfer process quantitatively. In stationary SERRS spectroelectrochemistry the analysis of a set of spectra acquired at various potentials can assess the Nernstian character the electron transfer process, yielding its $E^{\circ \prime}$ value $[5,14,19]$. Moreover, a time-resolved approach based on potential-jump experiments can give information about the electron transfer kinetic parameters $[5,56]$.

However, despite the broad potential window investigated (from $+0.15 \mathrm{~V}$ to $-1.0 \mathrm{~V}$ ) the electrochemical approach appeared to be unsuccessful in reducing the immobilized CYP2D6 completely, and failed to generate any reduced active enzyme at all. In fact, although there is some variability in the spectral data obtained from distinct electrodes, a common feature is the persistence of an intense marker band for the oxidized enzyme at $1,371 \mathrm{~cm}^{-1}$ even at potentials as negative as $-1.0 \mathrm{~V}$, together with the appearance of the marker band for a reduced inactive enzyme at $1,360 \mathrm{~cm}^{-1}$ (Fig. 5). An additional feature in the SERRS spectra of reduced CYP2D6 is the lack of any influence of DX (see supplementary material), contrary to what is observed with chemical reduction.

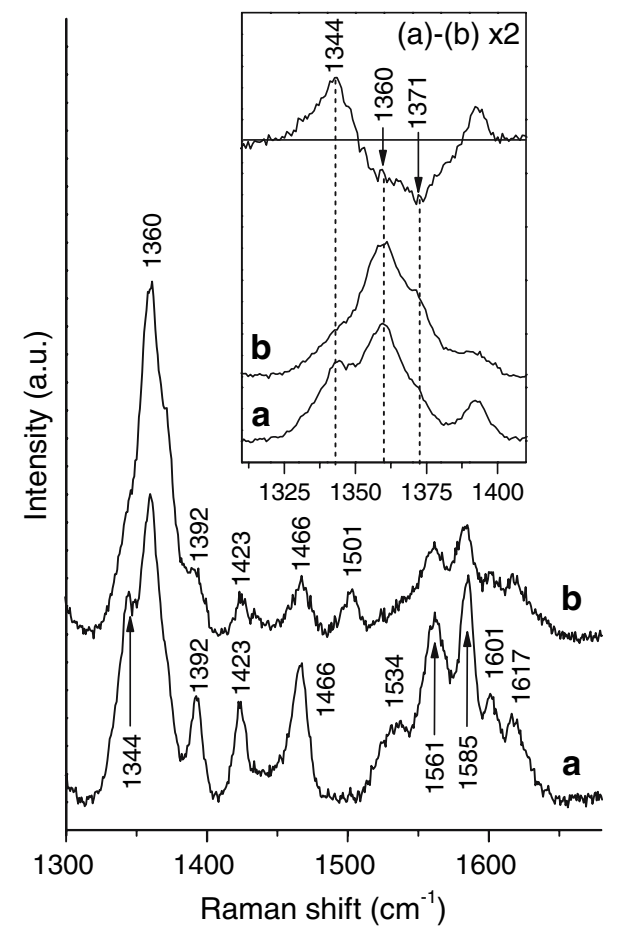

Fig. 4 SERRS spectra of CYP2D6 on MUA-coated Ag electrodes in the presence of buffered solution with dithionite and DX $(a)$ and without DX $(b)$. The inset shows the oxidation marker band regions in detail, together with their spectral difference. Experimental details are given in "Materials and methods"
Although the $E^{\circ}$ of CYP2D6 on MUA-coated Ag electrodes might differ significantly from the $E^{\circ \prime}$ reported for a polyaniline-doped glassy carbon electrode $(-0.12 \mathrm{~V})$ $[1,51]$, the persistence of a consistent amount of oxidized enzyme at $-1.0 \mathrm{~V}$ suggests an incomplete electron transfer. Despite the large variability of the $E^{\circ \prime}$ values found in the literature, depending on the CYP and on the method employed, no $E^{\circ \prime}$ is reported below $-0.6 \mathrm{~V}$ [1]. Consistent with this fact, CYP2D6 is effectively and completely reduced by a $5 \mathrm{mg} \mathrm{mL}^{-1}$ dithionite solution having a calculated redox potential of approximately $-0.7 \mathrm{~V}$ [57]. Therefore, on a pure thermodynamical basis, the application of a potential more negative than $-0.7 \mathrm{~V}$ to the $\mathrm{Ag}$ electrode should lead to a reduction of the adsorbed enzyme. The marked difference in the amount of reduced species obtained with chemical and electrochemical reduction (Fig. 6) might therefore be due to slower kinetics of the electron transfer process when using the second method.

The efficiency of the electrochemical reduction via the electrode is likely to be dependent on both the distance and

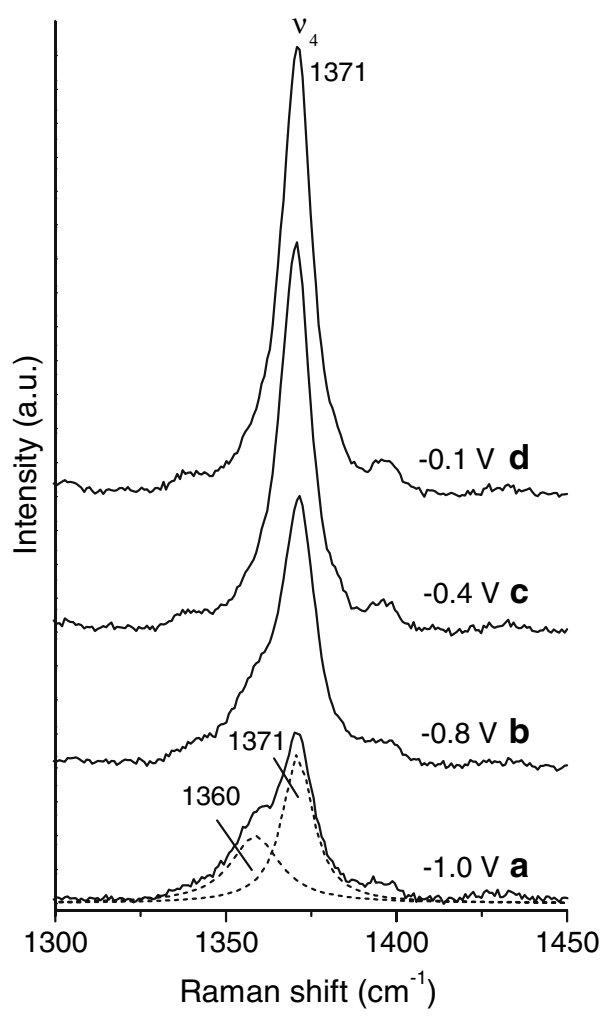

Fig. 5 Oxidation marker band $\left(v_{4}\right)$ regions of SERRS spectra of CYP2D6 on MUA-coated Ag electrodes at $-1.0 \mathrm{~V}(a),-0.8 \mathrm{~V}(b)$, $-0.4 \mathrm{~V}(c)$ and $-0.1 \mathrm{~V}(d)$ (vs. SCE), in the presence of buffer. The spectrum at $-1.0 \mathrm{~V}$ is fitted with Lorentzian functions for the reduced and oxidized forms, represented as dotted lines and labeled with their Raman shift $\left(1,371 \mathrm{~cm}^{-1}\right.$ for the oxidized form and $1,360 \mathrm{~cm}^{-1}$ for the reduced inactive P420 form). Experimental details are given in "Materials and methods" 
the orientation of the adsorbed protein with respect to the electrode surface $[1,3]$, whereas these two factors probably do not influence the dithionite reduction process. In vivo, electron transfer between the electroactive heme center, deeply buried inside the protein structure, and the electrondonating reductase occurs via an interaction involving specific domains on the protein surfaces [1, 3], with a strictly defined orientation. Conversely, the electron transfer from dithionite in solution will not be sensitive to the protein orientation, considering the capacity of the reducing agent to approach the enzyme from more directions. Moreover, while the minimum distance between the enzyme active site and the electrode surface is equivalent to the thickness of the MUA coating (approximately $19 \AA$ [22]) plus the heme-protein surface distance, dithionite is free to diffuse closer to the enzyme redox center. Therefore, an unfavorable enzyme orientation with respect to the coated metal surface or a too large heme-electrode distance would be consistent with experimental data, since it would slow down or suppress the electron transfer from the electrode, while dithionite would still be able to effectively reduce the CYP2D6 heme. Moreover, the inability of the electrochemical approach to generate any amount of reduced enzyme in its active form, which is otherwise observed in dithionite-reduced protein samples, suggests

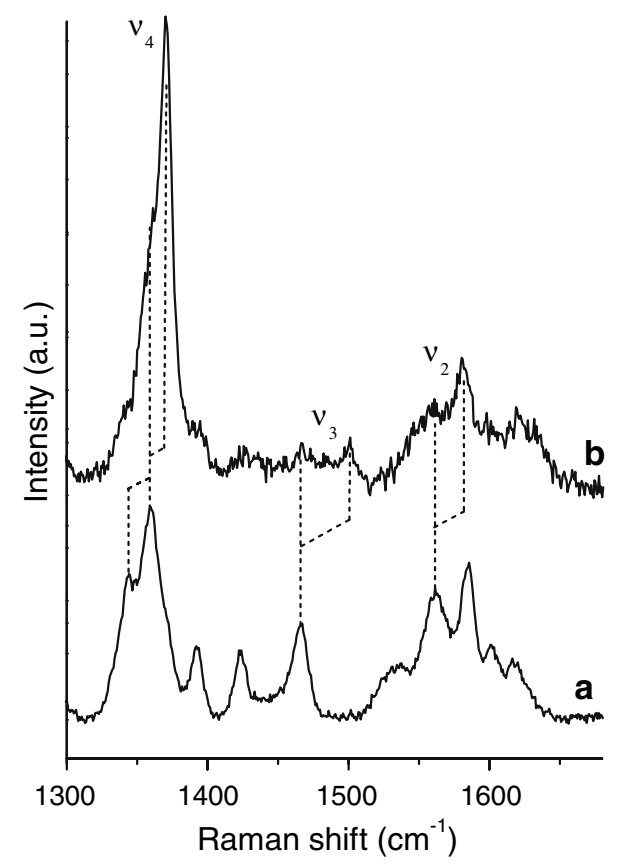

Fig. 6 Comparison between SERRS spectra of CYP2D6 on MUAcoated Ag electrodes $a$ chemically reduced with dithionite (open circuit) and $b$ at $-800 \mathrm{mV}$ (vs.SCE). Both spectra were recorded in the presence of DX. Marker bands for oxidation $\left(v_{4}\right)$ and $\operatorname{spin}\left(v_{3}, v_{2}\right)$ states are indicated. Experimental details are given in "Materials and methods" that CYP2D6 inactivation is triggered by the application of potentials between -0.4 and $-1.0 \mathrm{~V}$ during the enzymereduction attempts.

The intense electric field present at the interface of the SAM-coated Ag electrodes, which has recently been indicated as the main reason for the extensive conversion of the immobilized bacterial CYP101 to its inactive P420 forms [20], might be the cause of this potential-induced inactivation. However, according to the theory originally proposed by Smith and White [58] and later adapted by Murgida and Hildebrandt [22] and Lecomte et al. [59] to explain the behavior of $c$-type cytochromes adsorbed on electrodes, the intensity of this interfacial electric field is expected to decrease as the applied potential approaches the potential of zero charge $(-0.97 \mathrm{~V}$ for a polycrystalline Ag electrode) [13, 60-62]. Therefore, supposing that this theoretical model could be extended to CYPs (as it has been assumed in the case of CYP101 [20]), the interfacial electric field should be more intense at $-0.1 \mathrm{~V}$ than at $1.0 \mathrm{~V}$, whereas CYP2D6 inactivation occurs at more negative potentials, suggesting that in this case the interfacial electric field intensity as such is not responsible for the enzyme conversion to the $\mathrm{P} 420$ form upon electrochemical reduction. This hypothesis would be compatible with the electric-field-induced inactivation reported for CYP101 upon immobilization [20], considering that CYP101 is a soluble cytosolic enzyme, while CYP2D6 is a peripheral membrane protein, and the same interfacial electric field might have a very different effect on their respective active-site structures.

Besides tentatively excluding the interfacial electric field intensity as a cause, SERRS data alone cannot support any explanation for the CYP2D6 potential-induced inactivation. Additional detailed structural data on the secondary and tertiary structures of the adsorbed protein and on the nature and position of the amino acid residues interacting with the MUA monolayer would be highly valuable, and they might be retrieved in further studies using SERRS in combination with surface-enhanced infrared absorption spectroscopy [63] as well as other techniques [64-66].

\section{Conclusions}

Upon immobilization on MUA-coated Ag electrodes, the human enzyme CYP2D6 retains its active-site structure, its ability to reversibly bind a substrate (DX) and an exogenous heme ligand (imidazole), and its capacity to exchange electrons, demonstrating the efficiency of the coating to prevent major protein conformational changes or denaturation. However, despite its success in preserving an intact protein structure, the MUA coating appears to be unsuitable for performing direct electrochemistry experiments on 
immobilized CYP2D6, since its reduced state could not be adequately achieved by varying the electrode potential. The application of potentials from -0.4 to $-1.0 \mathrm{~V}$ appears to induce enzyme inactivation instead. Among other factors influencing the redox activity, a more favorable enzyme orientation or distance with respect to the electrode surface might be achieved using other coatings, an approach currently under study in our group.

In general, SERRS spectroscopy on coated Ag surfaces was proven to be successful in monitoring the active-site structure of immobilized CYP2D6, enabling the detection of reversible substrate and ligand binding using only very low amounts of protein. Besides its technological relevance as an optical biosensing tool, this technique might have a significant potential for fundamental research on the mechanisms of catalysis for CYPs, as well as for other heme enzymes.

Acknowledgements The authors thank Jeroen Lastdrager for helping in enzyme expression and purification, Joost Buijs for his technical assistance and Edwin Stigter and Robin Rozenhart for their help in preliminary experiments.

\section{References}

1. Bistolas N, Wollenberger U, Jung C, Scheller FW (2005) Biosens Bioelectron 20:2408-2423

2. Bernhardt R (2006) J Biotechnol 124:128-145

3. Shumyantseva VV, Bulko TV, Archakov AI (2005) J Inorg Biochem 99:1051-1063

4. Scheller FW, Wollenberger U, Lei C, Jin W, Ge B, Lehmann C, Lisdat F, Fridman V (2002) Rev Mol Biotechnol 82:411-424

5. Murgida DH, Hildebrandt P (2005) Phys Chem Chem Phys 7:3773-3784

6. Tian ZQ (2005) J Raman Spectrosc 36:466-470

7. Smith WE (1993) Methods Enzymol 226:482-495

8. Cotton TM, Kim JH, Chumanov GD (1991) J Raman Spectrosc 22:729-742

9. Zhou ZH, Wang GY, Xu ZZ (2006) Appl Phys Lett 88:3410434107

10. Bizzarri AR, Cannistraro S (2002) Appl Spectrosc 56:1531-1537

11. Kneipp K, Wang Y, Dasari RR, Feld MS (1995) Appl Spectrosc 49:780-784

12. Delfino I, Bizzarri AR, Cannistraro S (2005) Biophys Chem 113:41-51

13. Murgida DH, Hildebrandt P (2004) Acc Chem Res 37:854-861

14. Bernad S, Soulimane T, Mehkalif Z, Lecomte S (2006) Biopolymers $81: 407-418$

15. Zheng JW, Zhou Q, Zhou YG, Lu TH, Cotton TM, Chumanov G (2002) J Electroanal Chem 530:75-81

16. Rivas L, Soares CM, Baptista AM, Simaan J, Di Paolo RE, Murgida DH, Hildebrandt P (2005) Biophys J 88:4188-4199

17. Rivas L, Murgida DH, Hildebrandt P (2001) J Mol Struct 565:193-196

18. Dick LA, Haes AJ, Van Duyne RP (2000) J Phys Chem B 104:11752-11762

19. Millo D, Bonifacio A, Ranieri A, Borsari M, Gooijer C, van der Zwan G (2007) Langmuir 23:4340-4345

20. Todorovic S, Jung C, Hildebrandt P, Murgida DH (2006) J Biol Inorg Chem 11:119-127
21. Love JC, Estroff LA, Kriebel JK, Nuzzo RG, Whitesides GM (2005) Chem Rev 105:1103-1169

22. Murgida DH, Hildebrandt P (2001) J Phys Chem B 105:15781586

23. Guengerich FP (2004) Drug Metab Rev 36:159-197

24. Bertilsson L, Dahl ML, Dalen P, Al-Shurbaji A (2002) Br J Clin Pharmacol 53:111-122

25. Zanger UM, Raimundo S, Eichelbaum M (2004) NaunynSchmiedeberg's Arch Pharmacol 369:23-37

26. Vermeulen NPE (2003) Curr Top Med Chem 3:1227-1239

27. Rodrigues AD, Rushmore TH (2002) Curr Drug Metab 3:289_ 309

28. Bonifacio A, Millo D, Gooijer C, Boegschoten R, van der Zwan G (2004) Anal Chem 76:1529-1531

29. Keizers PHJ, de Graaf C, de Kanter FJJ, Oostenbrink C, Feenstra KA, Commandeur JNM, Vermeulen NPE (2005) J Med Chem 48:6117-6127

30. Omura T, Sato R (1964) J Biol Chem 239:2370-2378

31. Millo D, Ranieri A, Koot W, Gooijer C, van der Zwan G (2006) Anal Chem 78:5622-5625

32. Roth E, Hope GA, Schweinsberg DP, Kiefer W, Fredericks PM (1993) Appl Spectrosc 47:1794-1800

33. Bonifacio A, Keizers PHJ, Commandeur JNM, Vermeulen NPE, Robert B, Gooijer C, van der Zwan G (2006) Biochem Biophys Res Commun 343:772-779

34. Bonifacio A, Keizers PHJ, Vermeulen NPE, Commandeur JNM, Gooijer C, van der Zwan G (2007) Langmuir 23:1860-1866

35. Pylypenko O, Schlichting I (2004) Annu Rev Biochem 73:9911018

36. Denisov IG, Makris TM, Sligar SG, Schlichting I (2005) Chem Rev 105:2253-2277

37. Hildebrandt P (1992) In: Ruckpaul K, Rein H (eds) Relationships between structure and function of cytochrome P-450: experiments, calculations, models, vol. 7. Akademie, Berlin, pp 166215

38. Bonifacio A, Groenhof AR, Keizers PHJ, de Graaf C, Commandeur JNM, Vermeulen NPE, Ehlers AW, Lammertsma K, Gooijer C, van der Zwan G (2007) J Biol Inorg Chem 12:645-654

39. Deng TJ, Proniewicz LM, Kincaid JR, Yeom H, Macdonald IDG, Sligar SG (1999) Biochemistry 38:13699-13706

40. Dawson JH, Andersson LA, Sono M (1982) J Biol Chem 257:3606-3617

41. Smith SJ, Munro AW, Smith WE (2003) Biopolymers 70:620 627

42. Podust LM, Poulos TL, Waterman MR (2001) Proc Natl Acad Sci USA 98:3068-3073

43. Verras A, Alian A, Ortiz De Montellano PR (2006) Protein Eng Des Sel 19:491-496

44. Zhang W, Ramamoorthy Y, Kilicarslan T, Nolte H, Tyndale RF, Sellers EM (2002) Drug Metab Dispos 30:314-318

45. Hildebrandt P, Greinert R, Stier A, Taniguchi H (1989) Eur J Biochem 186:291-302

46. Noble MA, Quaroni LG, Chumanov G, Turner KL, Chapman SK, Hanzlik RP, Munro AW (1998) Biochemistry 37:15799-15807

47. Hildebrandt P, Garda H, Stier A, Bachmanova GI, Kanaeva IP, Archakov AI (1989) Eur J Biochem 186:383-388

48. Kalsbeck WA, Ghosh A, Pandey RK, Smith KM, Bocian DF (1995) J Am Chem Soc 117:10959-10968

49. Marzocchi MP, Smulevich G (2003) J Raman Spectrosc 34:725736

50. Hudecek J, Hodek P, Anzenbacherova E, Anzenbacher P (2007) Biochim Biophys Acta 1770:413-419

51. Iwuoha EI, Wilson A, Howel M, Mathebe NGR, Montane-Jaime K, Narinesingh D, Gueseppi-Elie A (2004) Anal Lett 37:929-941

52. Wells AV, Li PS, Champion PM, Martinis SA, Sligar SG (1992) Biochemistry 31:4384-4393 
53. Hudecek J, Anzenbacherova E, Anzenbacher P, Munro AW, Hildebrandt P (2000) Arch Biochem Biophys 383:70-78

54. Champion PM, Gunsalus IC, Wegner GC (1978) J Am Chem Soc 100:3743-3751

55. Wang J, Stuehr DJ, Rousseau DL (1995) Biochemistry 34:70807087

56. Lecomte S, Wackerbarth $\mathrm{H}$, Soulimane T, Buse G, Hildebrandt $P$ (1998) J Am Chem Soc 120:7381-7382

57. Mayhew SG (1978) Eur J Biochem 85:535-547

58. Smith CP, White HS (1992) Anal Chem 64:2398-2405

59. Lecomte S, Hildebrandt P, Soulimane T (1999) J Phys Chem B 103:10053-10064
60. Oklejas V, Sjostrom C, Harris JM (2002) J Am Chem Soc 124:2408-2409

61. Weidinger IM, Murgida DH, Dong W-F, Möhwald H, Hildebrandt P (2006) J Phys Chem B 110:522-529

62. Valette G, Hamelin A (1973) J Electroanal Chem 45:301-319

63. Ataka K, Heberle J (2004) J Am Chem Soc 126:9445-9457

64. Xu J, Bowden EF (2006) J Am Chem Soc 128:6813-6822

65. Niki K, Hardy WR, Hill MG, Li H, Sprinkle JR, Margoliash E, Fujita K, Tanimura R, Nakamura N, Ohno H, Richards JH, Gray HB (2003) J Phys Chem B 107:9947-9949

66. Sagara T, Kubo Y, Hiraishi K (2006) J Phys Chem B 110:1655016558 